\section{BASES MORFOLÓGICAS DE CÓMO PUDO HABERSE ORIGINADO EL DIMORFISMO SEXUAL EN EL PASTO BÚFALO}

\section{MORPHOLOGICAL BASIS OF HOW THE SEXUAL DIMORPHISM COULD HAVE BEEN ORIGINATED IN BUFFALOGRASS}

\author{
Juan M. Martínez Reyna* \\ y Sigifredo Armendáriz Erives
}

Departamento de Fitomejoramiento, Universidad Autónoma Agraria Antonio Narro (UAAAN). 25315, Buenavista, Saltillo, Coah. México. Tel. y Fax 018444110220.

* Autor para correspondencia (jmarrey@uaaan.mx)

\section{RESUMEN}

El dimorfismo sexual es una característica del zacate Búfalo [Buchloe dactyloides (Nutt.) Engelm. Sin. Bouteloua dactyloides Columbus], especie dioica nativa de Norteamérica con potencial para ser usada como césped en México. Sus inflorescencias son tan diferentes que en un principio las plantas femeninas fueron clasificadas como especie diferente de las plantas masculinas. En este estudio se describen los cambios morfológicos que pudieron haberse presentado en una inflorescencia hermafrodita de un presunto ancestro, para luego dar origen al dimorfismo sexual que hoy presenta B. dactyloides. Para ello se hicieron estudios descriptivos de las diferencias en la morfología floral dentro de las mismas inflorescencias y entre las inflorescencias de dos híbridos interploidales trimonoicos y sus progenitores dioicos. Con base en estas evidencias morfológicas aquí se postulan los eventos que pudieron haber originado el dimorfismo sexual. Para transformarse en la inflorescencia femenina actual, la inflorescencia hermafrodita ancestral tuvo que sufrir los siguientes cambios: reducción del número de espiguillas por ramificación espigada; reducción del espacio entre dichas ramificaciones; alargamiento de las segundas glumas para cubrir totalmente las florecillas; prolongación de las nervaduras central y de las dos laterales para formar los tres ápices dentados que coronan el involucro y aparición de pubescencia en estas glumas; reducción de las primeras glumas; ensanchamiento de las segundas glumas para envolver la florecilla en su totalidad; esterilización masculina de la florecilla hermafrodita; y endurecimiento de las glumas. En cambio, la inflorescencia masculina no debió pasar por grandes transformaciones, ya que morfológicamente es muy similar a las inflorescencias de especies hermafroditas del género Bouteloua con las que esta especie está emparentada.

Palabras clave: Buchloe dactyloides, Sin. Bouteloua dactyloides, dioecia, dimorfismo, morfología.

\section{SUMMARY}

Sexual dimorphism is characteristic of Buffalograss [Buchloe dactyloides (Nutt.) Engelm. Syn. Bouteloua dactyloides Columbus], a dioecious species native to North America which potentially could be used as turfgrass in México. Its inflorescences are so different that at first female and male plants were classified as different species. In this study we describe the morphological changes that a putative ancestral hermaphrodite inflorescence might have undergone to give rise to the sexual dimorphism present today in $B$. dactyloides. This descriptive study was conducted to establish the differences in floral morphology within the same inflorescence and between inflorescences of two trimonoecious interploidal hybrids and their dioecious parents. Based on these morphological evidences, we thereby postulate the events that might have happened for giving rise to the sexual dimorphism. To change into the current female inflorescence, the ancestral hermaphrodite inflorescence had to undergo the following changes: reduction of the number of spikelets per spicate branch; reduction of the space between these ramifications; elongation of the second glume to enclose the floret; extension of the central and two side veins to form the three toothed apexes that crown the bur; appearance of pubescence in the second glume; reduction of the first glume and widening of the second glume to fully wrap the floret; male sterilization of hermaphrodite flower; and glume induration. Male inflorescence however did not undergo major changes, because morphologically it is very similar to inflorescences of related hermaphroditic species in genus Bouteloua.

Index words: Buchloe dactyloides, Syn. Bouteloua dactyloides, dioecy, dimorphism, morphology.

\section{INTRODUCCIÓN}

El zacate Búfalo es una gramínea dioica dimórfica. Sus inflorescencias son tan diferentes que en un principio las plantas hembra y macho fueron clasificadas como especies diferentes. En 1818 Nuttall describió la especie a partir de un espécimen estaminado y la nombró Sesleria dactyloides, y en 1855 Steudel nombró como Antephora axilliflora al espécimen pistilado; finalmente, en 1859 Engelmann describe al zacate Búfalo como una especie dioica bajo el nombre de Buchloe dactyloides (Schaffner, 1920). La dioecia es la expresión extrema de la diclinia (separación de sexos en plantas diferentes). En su análisis filogenético, Weiblen et al. (2000) revelaron dos diferentes orígenes de la dioecia en monocotiledóneas; en uno ocurre la transición evolutiva de la dioecia a partir del hermafroditismo, que involucra a la ginodioecia (flores pistiladas y hermafroditas en plantas separadas) como un estado intermedio, y en el otro la dioecia surge a partir de la monoecia, quizás a través de un ajuste divergente de la proporción del sexo de las flores.

Estudios teóricos de los patrones evolutivos de la monoecia-dioecia en zacate Búfalo sugieren que la dioecia resulta de la redistribución gradual hacia un tipo de inflorescencia en las formas monoicas, más que de la completa esterilización del sexo opuesto en un evento 
único (Quinn, 1991). La ventaja de la polinización cruzada y una subsecuente especialización sexual de la inflorescencia femenina parece ser el principal factor que ocasionó este sistema dimórfico. En esta especie no existen diferencias entre sexos cuando se consideran características morfológicas vegetativas, de crecimiento y de éxito competitivo. La distribución espacial de los plantas masculinas y femeninas en la naturaleza no está relacionada con el tipo de suelo, humedad y topografía (Quinn, 1991).

El género Bouteloua, en el que Kinney et al. (2007) incluyen al género monotípico Buchloe, se distribuye predominantemente en Norteamérica y está formado por 57 especies, de las cuales 13 han desarrollado espiguillas unisexuales. Dentro de este grupo se presentan varios grados de diclinia, que incluyen: andromonoecia (flores estaminadas y hermafroditas en la misma plantas), ginodioecia, monoecia (flores pistiladas y estaminadas en la misma planta), trimonoecia (flores pistiladas, estaminadas y hermafroditas en la misma planta) y dioecia. Estos autores indican que $B$. dactyilodes presenta dos formas de diclinia, dioecia y monoecia.

El zacate Búfalo es una especie nativa de Norteamérica con gran potencial para ser usada como césped, porque posee las características adaptativas y de calidad de césped adecuadas para las condiciones edafoclimáticas del norte de México (Hernández et al., 2007).

Según Stubbendieck et al. (2003), la inflorescencia pistilada consiste de espiguillas con una sola florecilla cuya lema posee tres nervaduras, agrupadas en pequeñas estructuras rígidas llamadas involucros que contienen a las espiguillas con glumas desiguales; la primera es muy reducida y la segunda está endurecida y tiene tres ápices dentados que coronan el involucro. Generalmente tiene de tres a cinco espiguillas por involucro y dos involucros por inflorescencia, ésta parcialmente incluida en las vainas abultadas de las hojas superiores. Las plantas estaminadas presentan una panícula de dos a tres ramificaciones espigadas de 5 a $15 \mathrm{~mm}$ de longitud, y hasta con 10 espiguillas. Cada espiguilla, de 4 a $5.5 \mathrm{~mm}$ de longitud, posee dos florecillas sésiles, imbricadas y alineadas en dos filas sobre un raquis. La lema es más grande que las glumas. Es de hacer notar que en esta especie la inflorescencia masculina es morfológicamente más parecida a la inflorescencia de las especies hermafroditas del género Bouteloua.

En 2009, mientras se realizaba un estudio morfológico con hibridos interploidales, se encontró que dos híbridos del cruzamiento diploide por tetraploide presentaban anormalidades en el desarrollo de las inflorescencias. El objetivo del presente estudio es proponer los cambios morfológicos que debieron haberse presentado en una inflorescencia hermafrodita de un presunto ancestro encontrado en Zacatecas, México, cambios que podrían haber dado origen al dimorfismo sexual que hoy presenta B. dactyloides.

\section{MATERIALES Y MÉTODOS}

Este estudio se hizo en Buenavista, Saltillo, Coahuila, México ( $25^{\circ} 22^{\prime}$ LN, $101^{\circ} 00^{\prime} \mathrm{LO}$, y $1742 \mathrm{msnm}$ ) cuya temperatura media anual es de $19.8^{\circ} \mathrm{C}$. Se utilizaron dos híbridos, producto de cruzamientos interploidales (híbridos interploidales) de zacate Búfalo, cuyas genealogías y sitios de colecta de los progenitores y sus niveles de ploidía se muestran en el Cuadro 1.

\section{Recolección de muestras}

Las inflorescencias de los híbridos y de los progenitores se recolectaron en agosto del 2009, de plantas establecidas en macetas de $25 \mathrm{~cm}$ de diámetro y $30 \mathrm{~cm}$ de altura, rellenadas con mezcla del sustrato Pro-Mix ${ }^{\circledR}$ y suelo en una proporción 1:1 (v/v), en las que originalmente se colocó una planta por maceta. Las plantas se fertilizaron semanalmente en una dosis de $3 \mathrm{~g} \mathrm{~L}^{-1}$ con la fórmula $20 \mathrm{~N}$ 10P-30K (Grofol $\left.{ }^{\circledR}\right)$ y se regaron periódicamente.

\section{Caracterización morfológica}

Las inflorescencias se observaron en un estereoscopio Carl Zeiss Stemi 2000-C®; posteriormente se separaron las ramificaciones espigadas e involucros, y finalmente se disectaron las diferentes partes de las espiguillas y florecillas. Se efectuaron estudios morfológicos descriptivos para establecer las diferencias en la morfología floral dentro de las mismas inflorescencias y entre híbridos y progenitores. Para las fotografías se utilizó una cámara digital Cannon G5 ${ }^{\circledR}$ adaptada al estereoscopio.

Cuadro 1. Genealogía, sitio de colecta y nivel de ploidía de híbridos y progenitores del pasto Búfalo [Buchloe dactyloides (Nutt.) Engelm.].

\begin{tabular}{lllc}
\hline Genotipo & \multicolumn{1}{c}{ Genealogía } & \multicolumn{1}{c}{ Sitio de colecta } & Nivel de ploidía \\
\hline 'RC10' & Progenitor hembra & Real de Catorce, SLP. & $2 \mathrm{x}$ \\
'B 56' & Progenitor macho & Concepción del Oro, Zac. & $4 \mathrm{x}$ \\
'241056-2' & Híbrido interploidal & Saltillo, Coach. & $3 \mathrm{x}$ \\
'241056-3' & Híbrido interploidal & Saltillo, Coach. & $3 \mathrm{x}$ \\
\hline
\end{tabular}




\section{RESULTADOS Y DISCUSIÓN}

\section{Progenitores}

Las inflorescencias de los progenitores ' $\mathrm{RC} 10$ ' (femenino) y de 'B56' (masculino) fueron normales.

Inflorescencia masculina (Progenitor 'B56'). Es una panícula formada por tres ramificaciones espigadas que incluyen de 8 a 10 espiguillas estaminadas y formadas por dos glumas desiguales, la primera reducida hasta un tercio de la longitud de la segunda gluma, y esta última presenta una nervadura central prominente; la primera gluma es con o sin nervadura central y de apariencia hialina. Las glumas protegen a dos florecillas, cada una formada por la lema, la palea y los órganos sexuales. La lema sobresale de las glumas, ya que la longitud de la segunda gluma es dos tercios de la longitud de la lema, y con una nervadura central prominente más dos laterales. La palea es de aspecto hialino y del mismo tamaño que la lema, pero sólo tiene dos nervaduras laterales. La palea envuelve a tres anteras (Figura 1A).

Inflorescencia femenina (Progenitor 'RC10'). Es una panícula formada por dos o tres involucros, éstos formados a su vez por dos espiguillas. Cada espiguilla se compone de dos glumas, la primera reducida o vestigial, y la segunda endurecida y con tres aristas, de las cuales la central es la más alargada. Esta segunda gluma se fusiona exclusiva y parcialmente con una segunda gluma de una espiguilla estéril reducida. Las aristas sobresalen a la lema. Ambas glumas son pubescentes y envuelven a una florecilla con una lema de tres nervaduras (la central es prominente), y las tres se prolongan para formar pequeñas aristas. La palea es del mismo tamaño que la lema, de aspecto hialino y con dos nervaduras laterales. La lema y la palea envuelven a un pistilo con dos estigmas plumosos (Figura 1B).

\section{Híbridos interploidales}

Los híbridos '241056-2' y '241056-3' presentaron algunas anormalidades en su inflorescencia, con un tipo de diclinia denominado trimonoecia, pues aunque predominantemente son plantas hembras también presentan inflorescencias masculinas y espiguillas hermafroditas. Si bien esta diclinia se encuentra presente en algunas especies del género Bouteloua ( Kinney et al., 2007), para $B$. dactyloides es la primera vez que se reporta (Figura 2A). En las Figuras $2 \mathrm{~B}, 2 \mathrm{C}$ y $2 \mathrm{D}$ se muestran acercamientos de las inflorescencias: masculina, masculina con espiguillas hermafroditas, y femenina, respectivamente.

La inflorescencia masculina presenta modificaciones que se ilustran en la Figura 3. En conjunto, dichas modificaciones son una progresión hacia la inflorescencia femenina. En la Figura 3A (Izq.) se muestra una ramificación espigada del híbrido '241056-3' con tres espiguillas, y se compara con las últimas tres espiguillas de una ramificación espigada de 'B56' (Figura 3B.). Las espiguillas se separaron y se muestran en posición inversa en la Figura 3B; la de arriba es la espiguilla apical de la ramificación espigada.

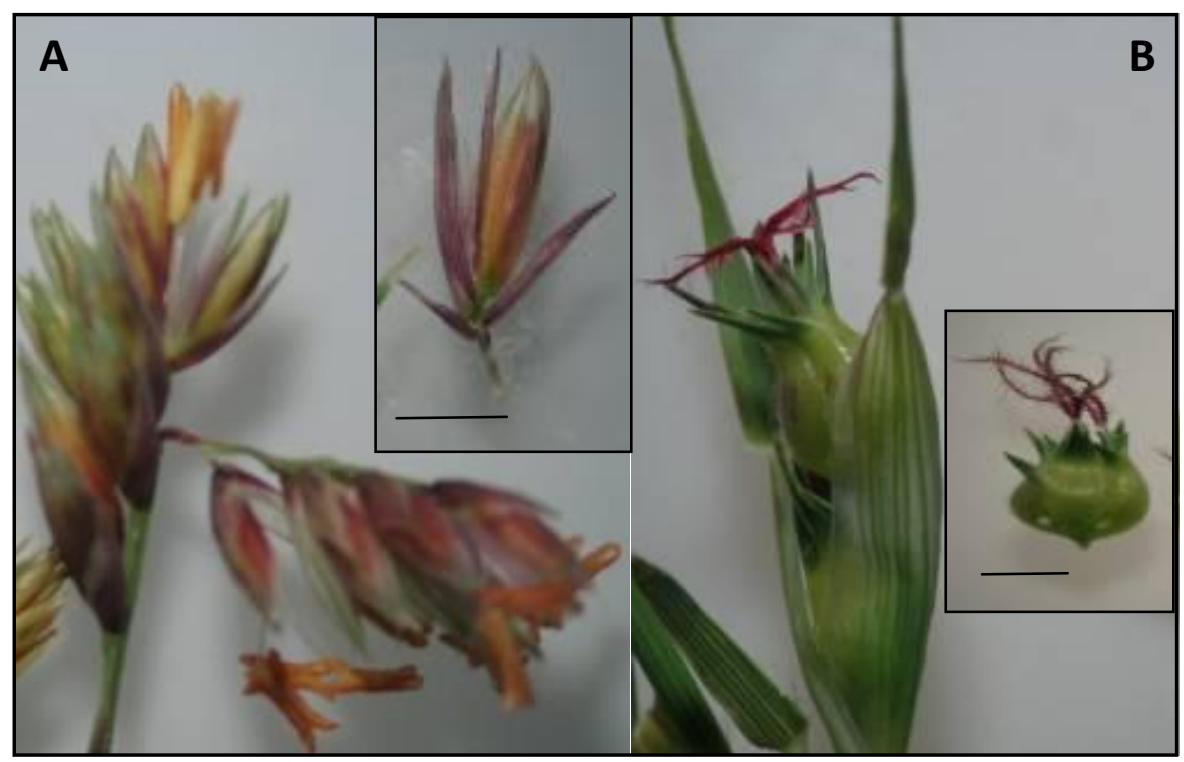

Figura 1. Inflorescencias de progenitores del pasto Búfalo. A) Inflorescencia masculina, con detalle de la espiguilla en recuadro; B) Inflorescencia femenina, con detalle del involucro en el recuadro. Escala $=2.5 \mathrm{~mm}$. 


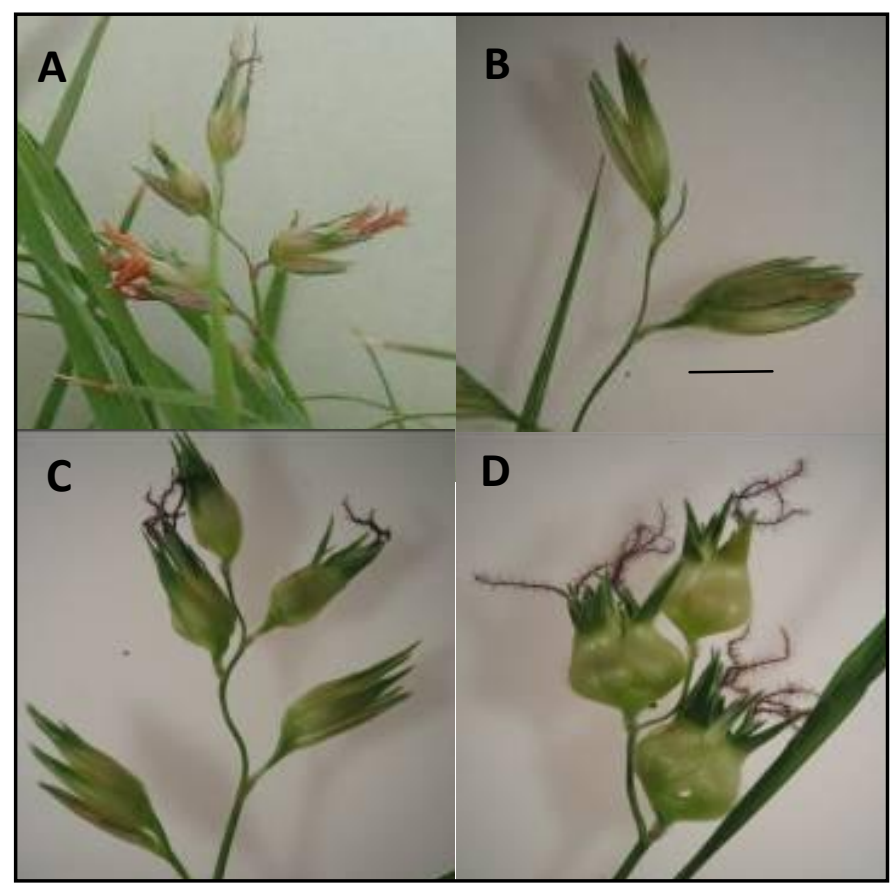

Figura 2. Inflorescencias de híbridos interploidales del pasto Búfalo. A) Planta trimonoica; B) Inflorescencia masculina; C) Inflorescencia masculina con espiguillas hermafroditas; D) Inflorescencia femenina. Escala $=2.5 \mathrm{~mm}$

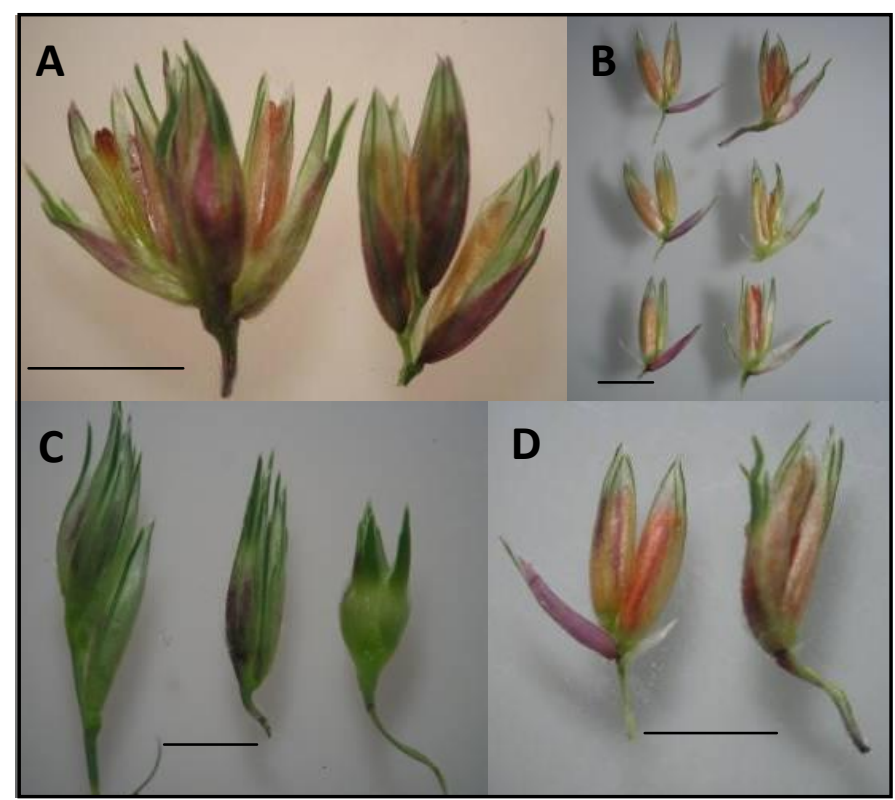

Figura 3. Modificaciones en espiguillas masculinas del híbrido '2410563' del pasto Búfalo. A) Ramificación espigada del híbrido (izq.), y espiguillas apicales de la ramificación espigada del progenitor 'B56'; B) Espiguillas masculinas de 'B56' (izq.) y del híbrido (der.); C) Ramificaciones espigadas de 'B56' (izq.), del híbrido (centro) e involucro del progenitor 'RC10' (der.); D) Acercamiento de espiguillas masculinas de 'B56' (izq.) y del híbrido (der.). Escala $=2.5 \mathrm{~mm}$.

Algunos cambios que se pueden apreciar en el híbrido con respecto al progenitor, son: en el híbrido la segunda gluma es tan grande como la lema y la primera gluma se va reduciendo hacia la espiguilla apical; la nervadura central de la gluma se prolonga sobre el ápice y empieza a formar una arista, y las dos nervaduras laterales empiezan a ser notorias. También se aprecia que la segunda gluma de la espiguilla apical se endurece y presenta pubescencia (Figura 3D). La reducción en el número de espiguillas de la ramificaron espigada del híbrido se muestra en el centro de la Figura 3C. Todas éstas son características de las espiguillas femeninas.

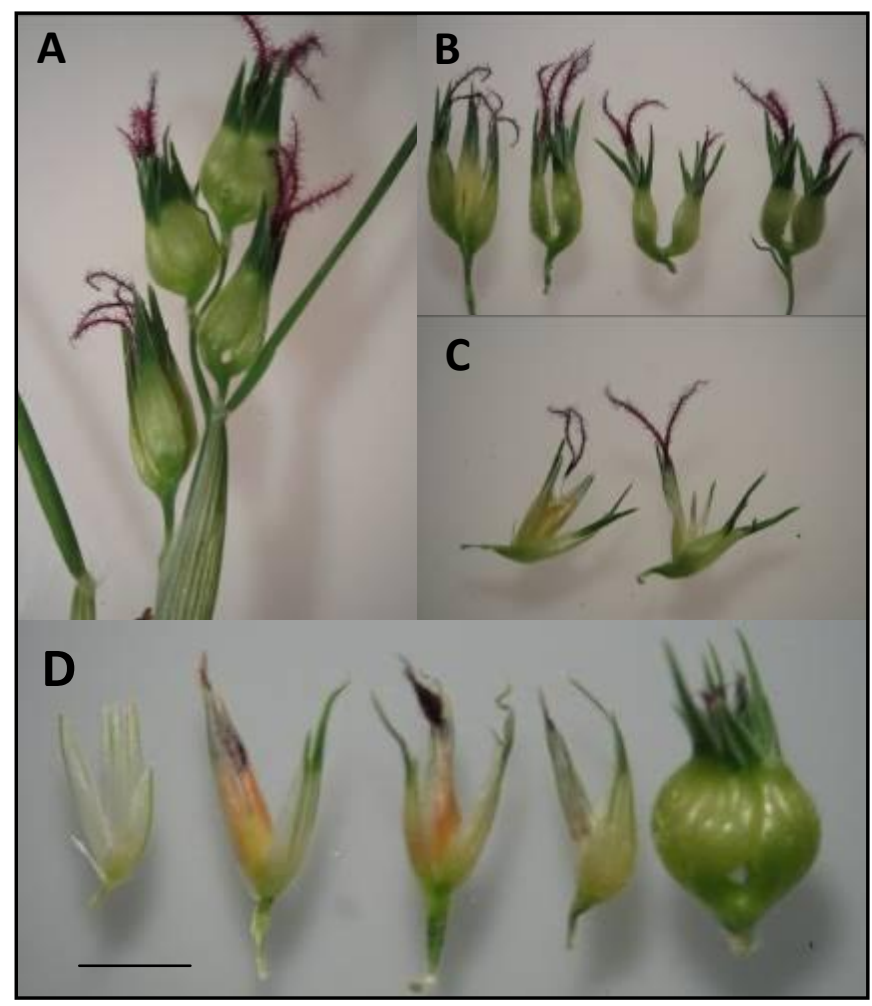

Figura 4. Modificaciones en espiguillas, involucros $y$ espiguillas hermafroditas del híbrido '241056-2' del pasto Búfalo. A) Inflorescencia de tipo masculino con espiguillas hermafroditas e involucros; B) Ramificaciones espigadas, en donde los extremos corresponden a la ramificación basal (izq.) y a la apical (der.); C) Acercamiento de los espiguillas una hermafrodita (izq.) y otra masculina (der.); D) Progresión de cambios desde la espiguilla masculina (izq.) hasta el involucro femenino (der.). Escala $=\mathbf{2 . 5} \mathbf{~ m m}$.

La inflorescencia con espiguillas morfológicamente hermafroditas, se puede describir como un fase intermedia entre las inflorescencias masculina y femenina que se encuentran en plantas dioicas (Figura 4A). En la Figura 4B, a la derecha se colocó la ramificación apical que ya presenta la forma del involucro característico de la inflorescencia femenina. Un acercamiento de las espiguillas hermafroditas y femeninas se presenta en la Figura 4C. Una progresión desde una espiguilla masculina hasta un involucro femenino (extremos) se muestra en la Figura 4D. La segunda espiguilla (de izquierda a derecha) es hermafrodita y muestra un 
alargamiento de la nervadura central en la segunda gluma, que forma la arista, y la primera gluma está reducida, lo que se hace aún más evidente en las siguientes espiguillas; en la tercera espiguilla, también hermafrodita, se aprecia la fusión parcial de la segunda gluma de una espiguilla estéril así como pubescencia de las glumas; en la cuarta espiguilla se observa el ensanchamiento de la segunda gluma pubescente que envuelve totalmente a una florecilla femenina, y que empieza a endurecerse.

En la aparición de esta última característica en la inflorescencia femenina, el Bisonte americano pudo haber sido un factor importante de distribución de la especie, pues las áreas de migración que tuvo este rumiante se sobrepone por completo con la zonas de distribución del zacate Búfalo en Norteamérica, por lo que es de suponerse que este pasto constituyó un componente importante en su alimentación (Quinn et al., 1994); el paso de los involucros por el tracto digestivo de estos animales debió haber favorecido la selección de los involucros más duros, para sobrevivir a los ácidos gástricos del rumen.

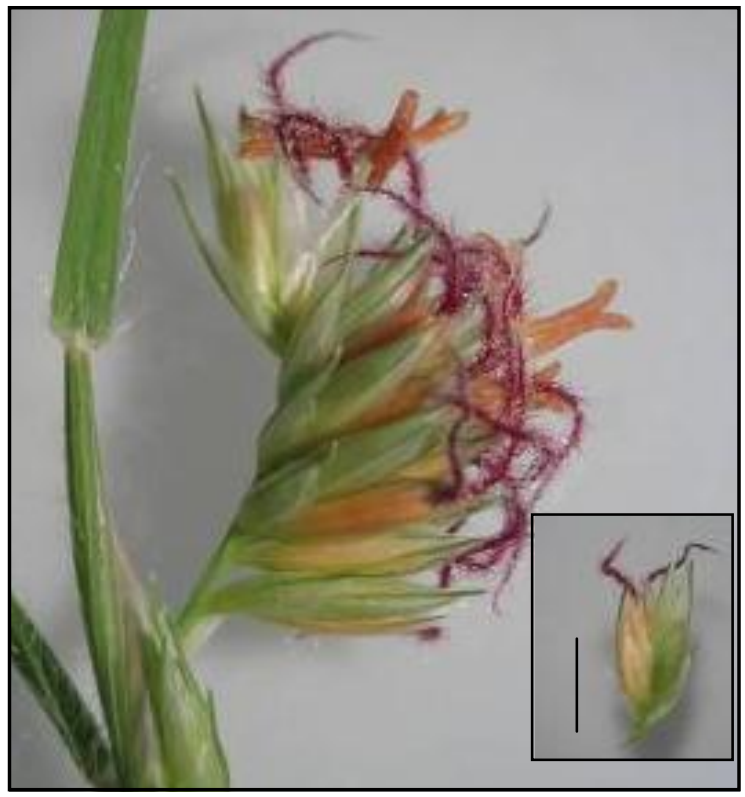

Figura 5. Inflorescencia hermafrodita del pasto Búfalo, con detalle de la espiguilla en el recuadro. Escala $=2.5 \mathrm{~mm}$.

\section{CONCLUSIONES}

Con base en estas evidencias morfológicas florales se pueden postular los eventos que dieron origen al dimorfismo sexual de $B$. dactyloides. La inflorescencia hermafrodita del probable ancestro, que se presume era similar a la que se muestra en la Figura 5 y que se encontró como una rareza en un material colectado en
Zacatecas, debió haber sufrido las siguientes modificaciones que la llevaron a transformarse en la inflorescencia femenina actual: reducción del número de espiguillas por ramificación espigada; reducción del espacio entre dichas ramificaciones; alargamiento de las segundas glumas para así cubrir totalmente a las florecillas; prolongación de las nervaduras central y de las dos laterales para formar los tres ápices dentados que coronan el involucro; aparición de pubescencia en estas glumas; reducción de las primeras glumas hasta convertirse en vestigiales en las espiguillas femeninas actuales; ensanchamiento de las segundas glumas para envolver totalmente a la florecilla; esterilización masculina de la florecilla hermafrodita; y endurecimiento de las glumas. En cambio, la inflorescencia masculina no sufrió grandes transformaciones porque es morfológicamente muy similar a inflorescencias de especies hermafroditas del género Bouteloua con las que esta especie está emparentada.

\section{BIBLIOGRAFÍA}

Hernández C R E, J M Martínez R, M H Reyes V, J R González D, H Díaz $S$ (2007) Caracterización morfológica y de calidad de césped de ecotipos de zacate Búfalo [Buchloe dactyloides (Nutt.) Engelm.]. Rev. Fitotec. Mex. 30:381-390.

Kinney M S, T Columbus, E A Friar (2007) Dicliny in Bouteloua (Poaceae: Chloridoideae): implications for evolution of dioecy. Aliso 23:605-614.

Quinn J A (1991) Evolution of dioecy in Buchloe dactyloides (Gramineae): tests for sex-specific vegetative characters, ecological differences and sexual niche-partitioning. Amer. J. Bot. 78:481-488.

Quinn J A, D P Mowrey, S M Emanuele, R B Whalley (1994) The "Foliage is the Fruit" Hypothesis: Buchloe dactyloides (Poaceae) and the Shortgrass Prairie of North America. Amer. J. Bot. 81:1545-1554.

Schaffner J H (1920) The diecious nature of buffalo-grass. Bull. Torrey Bot. Club 47:119-124.

Stubbendieck J, S L Hatch, L M Landholt (2003) North American Wildland Plants. A Field Guide. 6th ed. University of Nebraska Press. 501 p.

Weiblen G D, R K Oyama, M J Donoghue (2000) Phylogenetic analysis of dioecy in monocotyledons. Amer. Naturalist 155:4658. 